\title{
Layered Source-Channel Coding: A Distortion-Diversity Perspective
}

\author{
Sheng Jing, Lizhong Zheng and Muriel Médard \\ LIDS, MIT, Cambridge, MA 02139 \\ Email: \{sjing, lizhong, medard $\} @$ mit.edu
}

\begin{abstract}
Source-channel coding in time-varying channels without perfect side information at the transmitter suffers from uncertainty which may not always be averaged out. In channel coding, a main approach to address such uncertainty has been the outage formulation. In source coding, the main approaches to deal with such uncertainty have been multiple description coding (MDC) and successive refinement (SR). In this paper, we consider layered source-channel coding schemes relying on the MDC technique, originally proposed by Laneman et al, and the SR technique. We introduce the concept of distortion-diversity tradeoff, akin to the rate-diversity tradeoff, to consider the performance of these schemes. Our distortion-diversity perspective sheds some light on the performance comparison between various source-channel coding approaches in different operation regions.
\end{abstract}

\section{INTRODUCTION}

The nature of wireless channels, such as fading, shadowing and interference, can cause the channel quality to fluctuate over time. This time variation property of wireless channels induces uncertainty for communication, especially at the transmitter. If the time scale of channel variation is longer than the application delay constraints, then the channel quality uncertainty may not be averaged out. The classical approach to address this non-ergodic issue is the outage formulation of channel coding [1], which ensures a fixed level of reliability that the receiver is able to reconstruct the source to a certain level of accuracy. However, this type of schemes does not take advantage of good channel realizations. To allow for graceful degradation of the source reconstruction quality, several source coding techniques have been proposed in the application layer, including MDC [2] and SR [3]. To simultaneously address the channel uncertainty issue and the source reconstruction issue, we consider layered sourcechannel coding schemes, which have been recently proposed and studied (see [4], [5] and references therein). As the first step, we study two doublelayer source-channel coding schemes, which rely on the MDC technique [4] and the SR technique, respectively. We characterize the performance of each scheme through the achievable distortion distribution. Particularly, in the high resolution regime of the source and equivalently the high SNR regime of the channel, the distortion distribution can be cast as a tradeoff between the distortion exponent and the diversity order. Unlike the commonly adopted average distortion performance metric in [4], our proposed distortion-diversity perspective provides a more detailed performance comparison between various source-channel coding approaches in different operation regions.

The remaining paper is organized as follows: in Section II, we introduce the channel model and the outage formulation; in Section III, we describe several source coding techniques used in this paper; in Section IV, we analyze two source-channel coding schemes and characterize an outer bound; in Section $\mathrm{V}$, we provide performance comparison and conclude the paper.

\section{Channel Model \& Outage Formulation}

The channel model we consider is a discretetime parallel block fading channel, where the sender and the receiver are connected by a pair of AWGN channels subject to independent block Rayleigh fading. We only consider the single-block case in this paper, which is represented as follows,

$\mathbf{y}_{i}[n]=\mathbf{h}_{i} \mathbf{x}_{i}[n]+\mathbf{w}_{i}[n], i=1,2, n=1, \cdots, N$, 
where $N$ is the block length. The multiplicative fading coefficients $\mathbf{h}_{i}$ are i.i.d. $\mathcal{C N}(0,1)$ and the additive noise are i.i.d. $\mathcal{C N}(0,1)$. Channel state information (CSI) consists the instantaneous values of the fading coefficients. We assume no CSI at the transmitter (CSIT) while perfect CSI at the receiver (CSIR). The channel encoder maps a source message $i \in\{1, \cdots, M\}$ to a pair of length- $N$ channel input sequences, inducing a channel coding rate of $R_{c}=\frac{\ln M}{N}$. The input power constraint on each channel is SNR units of energy per channel use (SNR is also the transmit signal-to-noise-ratio), satisfied by each valid input sequence pair $\left(\underline{\mathbf{x}}_{1}, \underline{\mathbf{x}}_{2}\right)$ as follows,

$$
\frac{1}{N} \sum_{n=1}^{N}\left|x_{i}[n]\right|^{2} \leq \mathrm{SNR}, \quad i=1,2 .
$$

The channel decoder maps the pair of channel output sequences to $\hat{i} \in\{1, \cdots, M\}$.

Under the per-channel input power constraint of SNR with no CSIT and perfect CSIR, the singleblock supportable rate of parallel channel with fading coefficients $\mathbf{h}_{1}$ and $\mathbf{h}_{2}$ is defined to be

$$
I\left(\mathbf{h}_{1}, \mathbf{h}_{2}, \mathrm{SNR}\right) \triangleq \sum_{i=1,2} \ln \left(1+\left|\mathbf{h}_{i}\right|^{2} \mathrm{SNR}\right) .
$$

Note that the supportable rate (2) is a random variable rather than a constant, which depend on the fading coefficients only through their magnitudes. An outage event, denoted as $\mathcal{O}\left(\left|\mathbf{h}_{1}\right|,\left|\mathbf{h}_{2}\right|\right.$, SNR), happens if the channel coding rate $R_{c}$ exceeds the supportable rate. Accordingly, the outage probability is $\mathcal{P}\left[\mathcal{O}\left(\left|\mathbf{h}_{1}\right|,\left|\mathbf{h}_{2}\right|, \mathrm{SNR}\right)\right]$. In particular, we characterize the exponentially decaying speed of the outage probability w.r.t. SNR, namely the diversity order, which is defined as follows,

$\Delta_{\mathcal{O}\left(\left|\mathbf{h}_{1}\right|,\left|\mathbf{h}_{2}\right|, \mathrm{SNR}\right)}=-\lim _{\mathrm{SNR} \rightarrow \infty} \frac{\ln \mathcal{P}\left[\mathcal{O}\left(\left|\mathbf{h}_{1}\right|,\left|\mathbf{h}_{2}\right|, \mathrm{SNR}\right)\right]}{\ln \mathrm{SNR}}$

The diversity order is an important performance characteristic, especially in the high SNR regime. A convenient way to compute the diversity order is through the Laplace principle [1]: let

$$
\left|\mathbf{h}_{i}\right|^{2}=\mathrm{SNR}^{-\alpha_{i}}, \quad \alpha_{i}>0 ;
$$

re-formulate the outage event $\mathcal{O}\left(\left|\mathbf{h}_{1}\right|,\left|\mathbf{h}_{2}\right|, \mathrm{SNR}\right)$ as $\mathcal{O}^{\prime}\left(\alpha_{1}, \alpha_{2}\right)$ in the asymptotic of SNR; then the diversity order can be obtained by computing the dominant exponent sum as follows,

$$
\Delta_{\mathcal{O}\left(\left|\mathbf{h}_{1}\right|,\left|\mathbf{h}_{2}\right|, \mathrm{SNR}\right)}=\min _{\mathcal{O}^{\prime}\left(\alpha_{1}, \alpha_{2}\right)}\left(\alpha_{1}+\alpha_{2}\right) .
$$

The following form of outage event $\mathcal{O}^{\prime}\left(\alpha_{1}, \alpha_{2}\right)$ is particularly useful in this paper,

$$
\left\{\left(1-\alpha_{1}\right)^{+}+\left(1-\alpha_{2}\right)^{+}<\theta\right\},
$$

which is characterized in the following lemma.

Lemma II.1. Suppose that an outage event $\mathcal{O}^{\prime}\left(\alpha_{1}, \alpha_{2}\right)$ takes the form of (5). Then its diversity order is

$$
\Delta_{\mathcal{O}^{\prime}\left(\alpha_{1}, \alpha_{2}\right)}=2-\theta, \quad \theta \in(0,2] .
$$

Proof: The lemma can be verified by examining Figure 1, while the detailed proof is omitted for brevity.

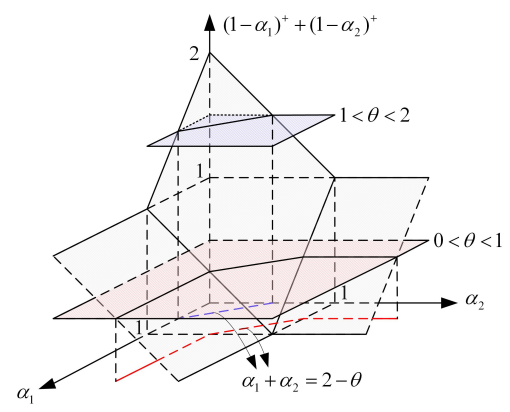

Fig. 1. Illustration of $\left(1-\alpha_{1}\right)^{+}+\left(1-\alpha_{2}\right)^{+}<\theta$

\section{SOURCE CODING}

We consider a source sequence $\underline{\mathbf{s}}=\left(\mathbf{s}_{1}, \cdots, \mathbf{s}_{n}\right)$ that is composed of independent and identically distributed (i.i.d.) random variables drawn from the same probability mass function (PMF) $p(s)$. The reconstructed source sequence is denoted as $\underline{\hat{\mathbf{s}}}=\left(\hat{\mathbf{s}}_{1}, \cdots, \hat{\mathbf{s}}_{n}\right)$. The source alphabet and the source reconstruction alphabet are $\mathcal{S}$ and $\hat{\mathcal{S}}$, respectively. In general, $\mathcal{S}$ and $\hat{\mathcal{S}}$ need not be the same. The symbol-wise distortion measure is a mapping $d: \mathcal{S} \times \hat{\mathcal{S}} \rightarrow \mathbb{R}$, and the sequence-wise distortion is defined as,

$d\left(\underline{s}^{n}, \underline{\hat{s}}^{n}\right)=\frac{1}{n} \sum_{i=1}^{n} d\left(s_{i}, \hat{s}_{i}\right), \quad(\underline{s}, \underline{\hat{s}}) \in \mathcal{S}^{n} \times \hat{\mathcal{S}}^{n}$. 


\section{A. Multiple Description Coding (MDC)}

An MDC quantizer is a mapping from the source sequence $\underline{\mathbf{s}}$ to two descriptions, $i_{1}$ and $i_{2}$,

$$
\phi_{n}: \mathcal{S}^{n} \rightarrow\left\{1, \cdots, M_{1}\right\} \times\left\{1, \cdots, M_{2}\right\} .
$$

The MDC reconstructor is composed of three separate reconstructors, namely the side reconstructors 1 and 2 and the central reconstructor. The side reconstructor 1 (resp. 2) uses the description $i_{1}$ (resp. $i_{2}$ ) only, while the central reconstructor uses both descriptions $\left(i_{1}, i_{2}\right)$,

$$
\begin{aligned}
\psi_{n, 1}: & \left\{1, \cdots, M_{1}\right\} \rightarrow \hat{\mathcal{S}}^{n}, \\
\psi_{n, 2}: & \left\{1, \cdots, M_{2}\right\} \rightarrow \hat{\mathcal{S}}^{n}, \\
\psi_{n, 0}: & \left\{1, \cdots, M_{1}\right\} \times\left\{1, \cdots, M_{2}\right\} \rightarrow \hat{\mathcal{S}}^{n} .
\end{aligned}
$$

The source description rates are $R_{s, i}=\frac{\ln M_{i}}{n}, i=$ 1,2 . The achieved distortion levels are

$D_{i}\left(\phi_{n}, \psi_{n, i}\right)=\mathcal{E}\left[d\left(\underline{\mathbf{s}}^{n}, \psi_{n, i}\left(\phi_{n}\left(\underline{\mathbf{s}}^{n}\right)\right)\right)\right], i=0,1,2$.

The quintuple $\left(R_{s, 1}, R_{s, 2}, D_{1}, D_{2}, D_{0}\right) \quad$ is achievable if there exists a sequence of $\left(R_{s, 1}, R_{s, 2}\right) \quad$ MDC quantizer-reconstructor quadruples $\left(\phi_{n}, \psi_{n, 1}, \psi_{n, 2}, \psi_{n, 0}\right)$ such that,

$$
\lim _{n \rightarrow \infty} D_{i}\left(\phi_{n}, \psi_{n, i}\right) \leq D_{i}, \quad i=0,1,2 .
$$

The MDC rate distortion region is the closure of all the achievable rate distortion quintuples $\left(R_{s, 1}, R_{s, 2}, D_{1}, D_{2}, D_{0}\right)$. Though the MDC rate distortion region has not been completely characterized for general sources and distortion measures, an achievable region has been provided in [6], which is recapped as follows.

Theorem III.1 (El-Gamal-Cover (EGC) Region [6]). Let the source PMF be $p(s)$ and the distortion measure $d(\cdot, \cdot)$ be bounded. An achievable rate region for the distortion triple $\left(D_{1}, D_{2}, D_{0}\right)$ is the convex hull of all rate pairs $\left(R_{s, 1}, R_{s, 2}\right)$ such that,

$$
\begin{aligned}
R_{s, 1} & >I(\mathbf{s} ; \mathbf{u}), \\
R_{s, 2} & >I(\mathbf{s} ; \mathbf{v}), \\
R_{s, 1}+R_{s, 2} & >I(\mathbf{s} ; \mathbf{u}, \mathbf{v})+I(\mathbf{u} ; \mathbf{v})
\end{aligned}
$$

for some joint PMF $p(s, u, v)=p(s) p(u, v \mid s)$ and reconstruction functions $\psi_{1}(\cdot), \psi_{2}(\cdot), \psi_{0}(\cdot, \cdot)$ such that,

$$
\begin{aligned}
& D_{1}=\mathcal{E} d\left(\mathbf{s}, \psi_{1}(\mathbf{u})\right) \\
& D_{2}=\mathcal{E} d\left(\mathbf{s}, \psi_{2}(\mathbf{v})\right) \\
& D_{0}=\mathcal{E} d\left(\mathbf{s}, \psi_{0}(\mathbf{u}, \mathbf{v})\right) .
\end{aligned}
$$

In particular, the symmetric rate distortion region is achieved by the following joint PMF $p(\mathbf{s}, \mathbf{u}, \mathbf{v})$ and reconstruction functions: the joint PMF $p(\mathbf{s}, \mathbf{u}, \mathbf{v})$ is induced by

$$
\begin{aligned}
& \mathbf{u}=\mathbf{s}+\mathbf{n}_{1}, \\
& \mathbf{v}=\mathbf{s}+\mathbf{n}_{2},
\end{aligned}
$$

where $\left(\mathbf{n}_{1}, \mathbf{n}_{2}\right)$ is independent of $\mathbf{s}$ and distributed as,

$$
\left(\mathbf{n}_{1}, \mathbf{n}_{2}\right) \sim \mathcal{C N}\left(\left[\begin{array}{l}
0 \\
0
\end{array}\right],\left[\begin{array}{cc}
\sigma^{2} & \rho \sigma^{2} \\
\rho \sigma^{2} & \sigma^{2}
\end{array}\right]\right) ;
$$

the reconstruction functions are

$$
\begin{aligned}
\psi_{1}(\mathbf{u}) & =\frac{1}{1+\sigma^{2}} \mathbf{u}, \\
\psi_{2}(\mathbf{v}) & =\frac{1}{1+\sigma^{2}} \mathbf{v}, \\
\psi_{0}(\mathbf{u}, \mathbf{v}) & =\frac{1}{2+(1+\rho) \sigma^{2}}(\mathbf{u}+\mathbf{v}),
\end{aligned}
$$

which achieves the following distortions,

$$
\begin{aligned}
D_{\text {partial }} & \triangleq D_{1}=D_{2}=\frac{\sigma^{2}}{1+\sigma^{2}}, \\
D_{\text {full }} & \triangleq D_{0}=\frac{(1+\rho) \sigma^{2}}{1+(1+\rho) \sigma^{2}} .
\end{aligned}
$$

Moreover, the rate constraints (7) simplify as follows,

$$
R_{s} \triangleq R_{s, 1}=R_{s, 2}>\ln \left(\frac{1}{\sqrt{1-\rho^{2}}} \frac{1+\sigma^{2}}{\sigma^{2}}\right) .
$$

The codebook generation, quantization and source reconstruction proceed as follows. First of all, two codebook $\mathcal{C}_{1}$ and $\mathcal{C}_{2}$ are constructed separately to include all rows of an $e^{n R_{s}} \times n$ matrix, the entries of which are generated i.i.d. according to $p(\mathbf{u})$ in (8) and $p(\mathbf{v})$ in (9), respectively. The two codebooks are then revealed to both the quantizer and the reconstructors. Secondly, given a source sequence $\underline{\mathbf{s}}$, the quantizer searches the codebooks $\mathcal{C}_{1}$ and $\mathcal{C}_{2}$ for a codeword pair $\left(\underline{\mathbf{u}}\left(i_{1}\right), \underline{\mathbf{v}}\left(i_{2}\right)\right)$ that is jointly 
typical with $\underline{\mathbf{s}}$ according to $p(\mathbf{s}, \mathbf{u}, \mathbf{v})$. The quantizer outputs $\left(i_{1}, i_{2}\right)$ as the quantization result (if multiple codeword pairs satisfy the joint typicality criterion, then the quantizer outputs the smallest index pair). The side and the central reconstructors output $\psi_{1}\left(\underline{\mathbf{u}}\left(i_{1}\right)\right), \psi_{2}\left(\underline{\mathbf{v}}\left(i_{2}\right)\right)$ and $\psi_{0}\left(\underline{\mathbf{u}}\left(i_{1}\right), \underline{\mathbf{v}}\left(i_{2}\right)\right)$ as the reconstructed source sequences, respectively.

\section{B. Successive Refinement (SR)}

A SR quantizer [3] maps the source sequence $\underline{\mathbf{s}}$ to two descriptions, $i_{b}$ and $i_{r}$, defined as follows,

$$
\phi_{n}: \mathcal{S}^{n} \rightarrow\left\{1, \cdots, M_{b}\right\} \times\left\{1, \cdots, M_{r}\right\} .
$$

The SR reconstructor is composed of two separate reconstructors, the base reconstructor and the refinement reconstructor. The base reconstructor uses the base description $i_{b}$ only, while the refinement reconstructor uses both descriptions $\left(i_{b}, i_{r}\right)$,

$$
\begin{aligned}
\psi_{n, b}: & \left\{1, \cdots, M_{b}\right\} \rightarrow \hat{\mathcal{S}}^{n}, \\
\psi_{n, r}: & \left\{1, \cdots, M_{b}\right\} \times\left\{1, \cdots, M_{r}\right\} \rightarrow \hat{\mathcal{S}}^{n} .
\end{aligned}
$$

The base description and refinement description rates are $R_{s, b}=\frac{\ln M_{b}}{n}$ and $R_{s, r}=\frac{\ln \left(M_{b} M_{r}\right)}{n}$, respectively. The achieved distortion levels by the base and the refinement reconstructors are

$$
\begin{aligned}
& D_{b}\left(\phi_{n}, \psi_{n, b}\right)=\mathcal{E}\left[d\left(\underline{\mathbf{s}}^{n}, \psi_{n, b}\left(\phi_{n}\left(\underline{\mathbf{s}}^{n}\right)\right)\right)\right], \\
& D_{r}\left(\phi_{n}, \psi_{n, r}\right)=\mathcal{E}\left[d\left(\underline{\mathbf{s}}^{n}, \psi_{n, r}\left(\phi_{n}\left(\underline{\mathbf{s}}^{n}\right)\right)\right)\right] .
\end{aligned}
$$

The rate distortion quadruple $\left(R_{b}, R_{r}, D_{b}, D_{r}\right)$ is achievable if there exists a sequence of rate- $\left(R_{s, b}, R_{s, r}\right)$ SR quantizer-reconstructor triples $\left(\phi_{n}, \psi_{n, b}, \psi_{n, r}\right)$ such that,

$$
\begin{gathered}
\lim _{n \rightarrow \infty} D_{b}\left(\phi_{n}, \psi_{n, b}\right) \leq D_{b}, \\
\lim _{n \rightarrow \infty} D_{r}\left(\phi_{n}, \psi_{n, r}\right) \leq D_{r} .
\end{gathered}
$$

The SR rate distortion region is the closure of all the achievable rate distortion quadruples $\left(R_{b}, R_{r}, D_{b}, D_{r}\right)$. Ideally, we want both the base layer rate distortion pair $\left(R_{b}, D_{b}\right)$ and the refinement layer rate distortion pair $\left(R_{r}, D_{r}\right)$ achieve the single description rate distortion boundary simultaneously. Reference [3] names this performance "successively refinable" and shows that the quadratic Gaussian sources are successively refinable. For a unit-variance complex gaussian source and squared-error distortion, two distortions levels $D_{b}$ and $D_{r}$ can be simultaneously achieved by the joint distribution of $\left(\mathbf{s}, \hat{\mathbf{s}}_{b}, \hat{\mathbf{s}}_{r}\right)$ induced by,

$$
\begin{aligned}
\mathbf{s} & =\hat{\mathbf{s}}_{r}+\mathbf{w}_{r}, \\
\hat{\mathbf{s}}_{r} & =\hat{\mathbf{s}}_{b}+\mathbf{w}_{b} .
\end{aligned}
$$

where $\hat{\mathbf{s}}_{b}, \mathbf{w}_{b}, \mathbf{w}_{r}$ are independently distributed as $\mathcal{C N}\left(0,1-D_{b}\right), \mathcal{C N}\left(0, D_{b}-D_{r}\right)$ and $\mathcal{C N}\left(0, D_{r}\right)$, resp. The codebook generation, quantization and reconstruction are as follows. First of all, a base codebook $\mathcal{C}_{b}$ is constructed to include all rows of an $e^{n R_{b}} \times n$ complex matrix, the entries of which are i.i.d. generated from $p\left(\hat{\mathbf{s}}_{b}\right)$ as defined in (14). For each codeword $\underline{\hat{s}}_{b}(i) \in \mathcal{C}_{b}$, a refinement codebook $\mathcal{C}_{r}(i)$ is constructed to include all rows of an $e^{n\left(R_{r}-R_{b}\right)}$ complex matrix, the entries of which are i.i.d. generated from $p\left(\underline{\hat{\mathbf{s}}}_{r} \mid \underline{\hat{s}}_{b}(i)\right)$ as defined in (14). The base codebook and the refinement codebooks are revealed to both the quantizer and the reconstructors. Given a source sequence $\underline{\mathbf{s}}$, the quantizer searches the base codebook $\mathcal{C}_{b}$ for a base codeword $\underline{\hat{\mathbf{s}}}_{b}\left(i_{b}\right)$ that is jointly typical with $\underline{\mathbf{s}}$. To guarantee that at least one base codeword satisfies the joint typicality criterion, we set the base description rate to be $R_{b}\left(D_{b}\right)=\ln \frac{1}{D_{b}}$. The quantizer then searches the corresponding refinement codebook $\mathcal{C}_{r}\left(i_{b}\right)$ for a refinement codeword $\underline{\hat{\mathbf{s}}}_{r}\left(i_{r}^{\prime}\right)$ that is jointly typical

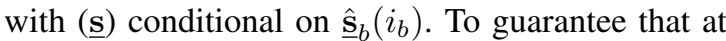
least one refinement codeword satisfies the conditional joint typicality, we set the parameter $R_{r}$ to be $R_{r}\left(D_{r}\right)=\ln \frac{1}{D_{r}}$. The quantizer outputs $\left(i_{b}, i_{r}\right)$, where $i_{r} \triangleq\left(i_{b}, i_{r}^{\prime}\right)$, as the quantization results (if multiple codeword pairs satisfy the above joint typicality criterion, the quantizer outputs the smallest pair). Given that the index pair $i_{b}, i_{r}$ is correctly received, the base reconstructor outputs $\underline{\hat{\mathbf{s}}}_{b}\left(i_{b}\right)$ and the refinement reconstructor outputs $\underline{\hat{\mathbf{s}}}_{r}\left(i_{r}^{\prime}\right) \in \mathcal{C}\left(i_{b}\right)$ as the reconstructed source sequence, respectively.

\section{SOURCE-CHANnEL SCHEMES}

We consider point-to-point communication between a sender and a receiver. The sender collects a length- $N$ source sequence $\underline{\mathbf{s}}$, maps it to length$N$ channel codewords $\underline{\mathbf{x}}_{1}$ and $\underline{\mathbf{x}}_{2}$ using a sourcechannel encoder and sends them over the parallel 
fading channel to the receiver. The receiver reconstructs the source sequence as $\underline{\hat{\mathbf{s}}}$ from the channel output sequences $\underline{\mathbf{y}}_{1}$ and $\underline{\mathbf{y}}_{2}$ using a source-channel decoder. In this paper, we consider the bandwidth expansion ratio 1 case only, equivalently $N$ source symbols sent over $N$ channel uses.

\section{A. MDC with Joint Decoding (MDC-JD)}

The MDC-JD scheme was proposed in [4] and is illustrated in in Figure 2.

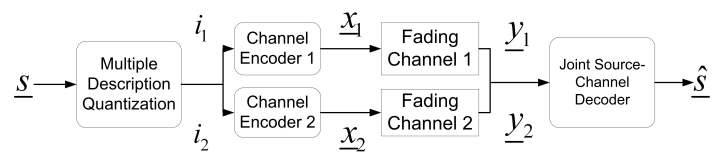

Fig. 2. MDC-JD Diagram

For the source coding part, the MDC-JD scheme employs the EGC quantizer. We consider the symmetric case only, where $R_{s}$ denotes the rate of each source description. The two descriptions from the EGC quantizer, $i_{1}$ and $i_{2}$, are separately encoded to channel input sequences $\underline{x}_{1}$ and $\underline{x}_{2}$. We use $R_{c}$ to denote the channel coding rate on each channel. Note that each description is associated with both a source quantization sequence and a channel codeword. Given the channel output sequences $\underline{y}_{1}$ and $\underline{\mathbf{y}}_{2}$, the receiver creates two lists, $\mathcal{L}_{1}$ and $\mathcal{L}_{2}$, of the possible source descriptions, $\hat{i}_{1}$ and $\hat{i}_{2}$ and the associated source quantization sequences, $\underline{\mathbf{u}}\left(\hat{i}_{1}\right)$ and $\underline{\mathbf{v}}\left(\hat{i}_{2}\right)$, respectively. The receiver searches $\overline{\mathcal{L}}_{1} \times \mathcal{L}_{2}$ for a source description pair $\left(\hat{i}_{1}, \hat{i}_{2}\right)$ such that $\left(\underline{\mathbf{u}}\left(\hat{i}_{1}\right), \underline{\mathbf{v}}\left(\hat{i}_{2}\right)\right)$ is jointly typical w.r.t. the joint distribution of $\mathbf{u}$ and $\mathbf{v}$, which is defined after Theorem III.1. If only one jointly typical source description pairs found, then the receiver obtains a "full" reconstruction with the central reconstructor $\psi_{0}(\cdot, \cdot)$. If more than one jointly typical source description pair is found, then the receiver checks the cardinalities of $\mathcal{L}_{1}$ and $\mathcal{L}_{2}$. If either $\left|\mathcal{L}_{1}\right|=1$ or $\left|\mathcal{L}_{2}\right|=1$, then the receiver obtains partial reconstruction using the side reconstructor $\psi_{1}(\cdot)$ or $\psi_{2}(\cdot)$. Note that the reconstructors $\psi_{0}, \psi_{1}$ and $\psi_{2}$ are specified after Theorem III.1.
To achieve a partial reconstruction distortion of $D_{\text {partial }}$ and a full reconstruction distortion of $D_{\text {full }}$, the EGC quantizer parameters $\sigma^{2}$ and $\rho$ are set according to (10) and (11) as follows,

$$
\begin{aligned}
\sigma^{2} & =\frac{D_{\text {partial }}}{1-D_{\text {partial }}} \\
\rho & =-1+\frac{2 D_{\text {full }} /\left(1-D_{\text {full }}\right)}{D_{\text {partial }} /\left(1-D_{\text {partial }}\right)} .
\end{aligned}
$$

The source description rate constraint (12) reduces to

$$
R_{s}>\frac{1}{2} \ln \frac{\left(1-D_{\text {full }}\right)^{2}}{4\left(1-D_{\text {partial }}\right) D_{\text {full }}\left(D_{\text {partial }}-D_{\text {full }}\right)}
$$

In the high SNR regime, we re-parameterize the distortion levels as

$$
D_{\text {partial }}=\mathrm{SNR}^{-d_{p}}, \quad D_{\text {full }}=\mathrm{SNR}^{-d_{f}},
$$

where $0<d_{p}<d_{f}$. Then the rate constraint (17) reduces to

$$
\lim _{\mathrm{SNR} \rightarrow \infty} \frac{R_{s}}{\ln \mathrm{SNR}} \geq \frac{d_{p}+d_{f}}{2},
$$

In the following, we assume equality holds in the above rate constraint. Moreover, the channel coding rate $R_{c}$ is the same as the source coding rate $R_{s}$ for the no bandwidth expansion case we are considering. The full reconstruction outage event, $\mathcal{O}_{\text {full }}\left(R_{c}, \mathbf{h}_{1}, \mathbf{h}_{2}, \mathrm{SNR}\right)$, is characterized in [4] as follows,

$$
\left\{\sum_{i=1,2}\left(R_{c}-I\left(\mathbf{h}_{i}, \mathrm{SNR}\right)\right)^{+}>I(\mathbf{u} ; \mathbf{v})\right\} .
$$

where $I(\mathbf{u} ; \mathbf{v})=\ln \frac{\left(1+\sigma^{2}\right)^{2}}{\left(1+\sigma^{2}\right)^{2}-\left(1+\rho \sigma^{2}\right)^{2}}$. With $\sigma^{2}$ and $\rho$ specified in (15) and (16), the above $I(\mathbf{u} ; \mathbf{v})$ expression reduces in the high SNR regime to

$$
\lim _{\mathrm{SNR} \rightarrow \infty} \frac{I(\mathbf{u} ; \mathbf{v})}{\ln \mathrm{SNR}}=d_{p} .
$$

The outage event of the partial reconstruction is

$$
\mathcal{O}_{\text {partial }}\left(R_{c}, \mathbf{h}_{1}, \mathbf{h}_{2}, \mathrm{SNR}\right)=\mathcal{O}_{1} \cap \mathcal{O}_{2} \cap \mathcal{O}_{\text {full }},
$$

where $\mathcal{O}_{i}$ is the event that channel $i$ 's output sequence is not sufficient to decode description $i$,

$\mathcal{O}_{i}\left(R_{c}, \mathbf{h}_{i}, \mathrm{SNR}\right)=\left\{R_{c}-I\left(\mathbf{h}_{i}, \mathrm{SNR}\right)>0\right\}, i=1,2$. 
We visualize the relationship between the outage events $\mathcal{O}_{i}, \mathcal{O}_{\text {full }}$ and $\mathcal{O}_{\text {partial }}$ in Figure 3. Note that $\mathcal{O}_{1}^{c} \cap \mathcal{O}_{2}^{c}$ implies $\mathcal{O}_{\text {full }}^{c}$.

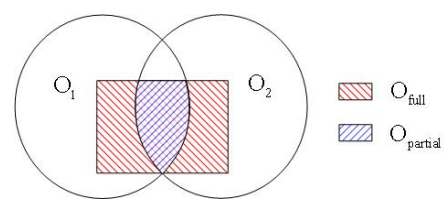

Fig. 3. Venn Diagram relating $\mathcal{O}_{1}, \mathcal{O}_{2}, \mathcal{O}_{\text {full }}$ and $\mathcal{O}_{\text {partial }}$

Lemma IV.1 (MDC-JD Diversity Orders). For the $M D C-J D$ scheme, the full reconstruction and the partial reconstruction diversity orders are as follows: for $0<d_{p}<d_{f}<2$,

$$
\begin{gathered}
\Delta_{\text {partial }}=2-d_{f}, \\
\Delta_{\text {full }}=\left\{\begin{array}{cl}
1-\left(d_{f}-d_{p}\right) / 2, & \text { if } d_{p}+d_{f}<2, \\
2-d_{f}, & \text { if } d_{p}+d_{f}>2 .
\end{array}\right.
\end{gathered}
$$

Proof: The proof is omitted for brevity.

Combining (18) with Lemma IV.1, we are able to characterize the distortion-diversity tradeoff of $\left(d_{p}, \Delta_{\text {partial }}, d_{f}, \Delta_{\text {full }}\right)$ on both layers achieved by the MDC-JD scheme. We visualize this distortiondiversity tradeoff along the direction of $\left(d_{p}, d_{f}\right)=$ $(d, \alpha d)$ in Figure 4 for several $\alpha$ values,

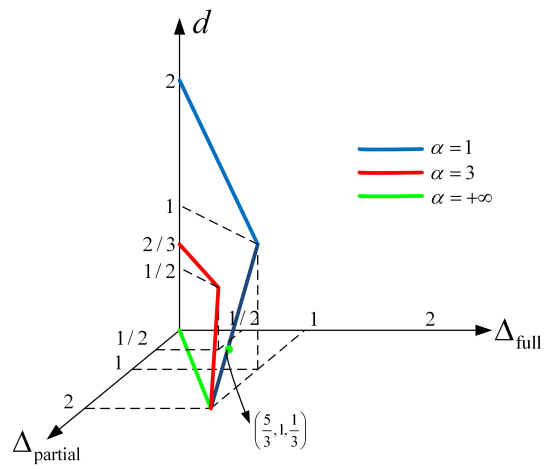

Fig. 4. Single-Block Distortion-Diversity Tradeoff of the MDCJD Scheme, $\left(d_{p}, d_{f}\right)=(d, \alpha d)$
Remark The parameter $\alpha$ controls the closeness between the two source quantization sequences $\mathbf{u}\left(i_{1}\right)$ and $\mathbf{v}\left(i_{2}\right)$. The $\alpha$ close to 1 case can be achieved by setting $\rho$ close to $0^{1}$, indicating that, in the high SNR regime, the two descriptions $\mathbf{u}\left(i_{1}\right)$ and $\mathbf{v}\left(i_{2}\right)$ are generated by adding two independent noise. This provides good partial reconstruction quality but no significant improvement for the full reconstruction. On the other hand, the large $\alpha$ case can be achieved by setting $\sigma^{2}$ close to 1 and $\rho$ close to -1 , indicating that, in the high SNR regime, the two source descriptions are generated by adding two nearly-perfectly-negatively-correlated noises of power level close to the source signal. This provides good full reconstruction but poor partial reconstruction.

The parameter $\alpha$ also affects the joint decoding reliability and the diversity orders. If $\alpha$ is close to 1 , then $I(\mathbf{u} ; \mathbf{v})$ achieves its maximum and therefore the joint decoder provides the most significant additional protection to the full reconstruction. However, if $\alpha$ is large, the joint decoder degenerates to become a pair of separate channel decoders.

Example: green point in Figure 4 The coordinates of this point are $(5 / 3,1,1 / 3)$, which correspond to $\alpha=1$ and $d=1 / 3$. The EGC quantizer parameters are set as follows,

$$
\sigma^{2}=\mathrm{SNR}^{-1 / 3}, \quad \rho=0 .
$$

According to (8) and (9), the two source descriptions are generated by adding independent Gaussian noise of power SNR ${ }^{-1 / 3}$ to the source sequence. The mutual information between the two source descriptions is $I(\mathbf{u} ; \mathbf{v})=1 / 3 \ln S N R$. The reconstruction functions $\psi_{0}, \psi_{1}$ and $\psi_{2}$ are the MMSE estimators of the source sequence. From (10) and (11), the partial reconstruction and the full reconstruction distortion levels are,

$$
\begin{aligned}
D_{\text {partial }}=\mathrm{SNR}^{-1 / 3} & \Rightarrow d_{\text {partial }}=1 / 3, \\
D_{\text {full }}=\mathrm{SNR}^{-1 / 3} & \Rightarrow d_{\text {full }}=1 / 3,
\end{aligned}
$$

Applying Lemma II.1, the outage events (20) and (22) reduce to Figure 5. If we proceed along the

${ }^{1}$ From (16), setting $\rho$ close to any constant in $(-1,1]$ also yields $\alpha=1$ 


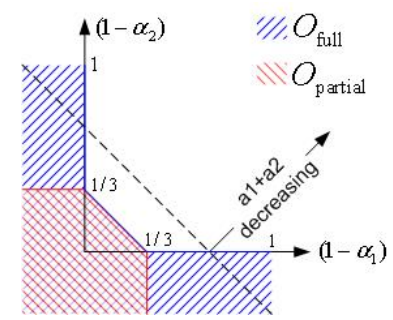

Fig. 5. Example: MDC-JD Outage Events Relationship

direction of decreasing $\alpha_{1}+\alpha_{2}$ in Figure 5, we can read off the smallest $\alpha_{1}+\alpha_{2}$ in each outage event as follows,

$$
\Delta_{\text {partial }}=5 / 3, \quad \Delta_{\text {full }}=1 .
$$

The green point is therefore achieved.

\section{B. SR with Superposition Coding (SR-SPC)}

A block diagram of the SR-SPC scheme is shown in Figure 6.

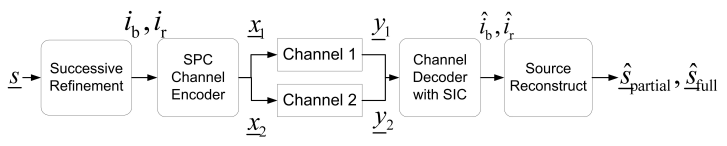

Fig. 6. SR-SPC Diagram

The sender quantizes a length- $N$ source sequence $\underline{\mathbf{s}}$ into two descriptions, $i_{b} \in\left\{1, \cdots, M_{b}\right\}$ and $i_{r} \in\left\{1, \cdots, M_{r}\right\}$, using a successive refinement source quantizer. The source description rates are $R_{s, b}=\frac{\ln M_{b}}{N}$ and $R_{s, r}=\frac{\ln M_{b}+\ln M_{r}}{N}$, respectively. Since the quadratic Gaussian source is successively refineable, the above source description rates achieve the following base layer and refinement layer distortion exponents,

$$
d_{b}\left(r_{b}, r_{r}\right)=r_{b}, \quad d_{r}\left(r_{b}, r_{r}\right)=r_{r}
$$

The two source descriptions are then encoded into the channel input sequences by a SPC channel encoder as follows: the sender splits the input power SNR between the two source descriptions as $P_{b}=\mathrm{SNR}-\mathrm{SNR}^{1-\beta}$ and $P_{r}=\mathrm{SNR}^{1-\beta},(\beta>0)$; two channel codebooks, $\mathcal{C}_{b}\left(M_{b}\right.$ codewords, each codeword being a $2 \times N$ matrix $)$ and $\mathcal{C}_{r}\left(M_{r}\right.$ codewords, each codeword being a $2 \times N$ matrix), are generated separately with entries i.i.d. according to $\mathcal{C N}\left(0, P_{b}\right)$ and $\mathcal{C N}\left(0, P_{r}\right)$, respectively; given the source description pair $\left(i_{b}, i_{r}\right)$, the sender transmits the sum of the $i_{b}^{\text {th }}$ codeword of $\mathcal{C}_{b}$ and the $i_{r}^{\text {th }}$ codeword of $\mathcal{C}_{r}$ through the parallel fading channel. The channel coding rates are $R_{c, b}=\frac{\ln M_{b}}{N}$ and $R_{c, r}=\frac{\ln M_{b}+\ln M_{r}}{N}$, respectively. Note that $R_{s, b}=$ $R_{c, b}$ and $R_{s, r}=R_{c, r}$, respectively. We let the base layer soruce/channel coding rate and the refinement layer source/channel coding rate scale with SNR as $R_{c, b}=r_{b} \ln \operatorname{SNR}\left(r_{b}>0\right)$ and $R_{c, r}=r_{r} \ln$ SNR $\left(r_{r}>r_{b}\right)$, respectively. At the receiver, the successive interference cancelation decoder proceeds as follows: searches for the base description codeword, treating the refinement codeword as noise; subtracts the decoded base description codeword; searches for the refinement codeword. Therefore, the singleblock supportable rate for the base layer is

$I_{b}\left(\beta, \mathbf{h}_{1}, \mathbf{h}_{2}, \mathrm{SNR}\right)=\sum_{i=1,2} \ln \left(1+\frac{\left|\mathbf{h}_{i}\right|^{2}\left(\mathrm{SNR}-\mathrm{SNR}^{1-\beta}\right)}{1+\left|\mathbf{h}_{i}\right|^{2} \mathrm{SNR}^{1-\beta}}\right)$,

where the refinement layer power $\mathrm{SNR}^{1-\beta}$ is counted as noise. Moreover, we have the following conditional single-block supportable rate for the refinement layer given that the base layer has been correctly decoded and subtracted,

$I_{r}\left(\beta, \mathbf{h}_{1}, \mathbf{h}_{2}, \mathrm{SNR}\right)=\sum_{i=1,2} \ln \left(1+\left|\mathbf{h}_{i}\right|^{2} \mathrm{SNR}^{1-\beta}\right)$.

Therefore, the base reconstruction outage event $\mathcal{O}_{b}\left(\beta, R_{c, b}, \beta, \mathbf{h}_{1}, \mathbf{h}_{2}, \mathrm{SNR}\right)$ is

$$
\left\{I_{b}\left(\beta, \mathbf{h}_{1}, \mathbf{h}_{2}, \mathrm{SNR}\right)<R_{c, b}\right\},
$$

and the refinement reconstruction outage event $\mathcal{O}_{r}\left(\beta, R_{c, b}, R_{c, r}, \beta, \mathbf{h}_{1}, \mathbf{h}_{2}, \mathrm{SNR}\right)$ is

$$
\mathcal{O}_{b} \bigcup\left\{I_{r}\left(\beta, \mathbf{h}_{1}, \mathbf{h}_{2}, \mathrm{SNR}\right)<R_{c, r}-R_{c, b}\right\} .
$$

Lemma IV.2 (SR-SPC Diversity Orders). The SR$S P C$ scheme achieves the following base layer and refinement layer diversity orders,

$$
\Delta_{b}\left(r_{b}, r_{r}\right)=\left(2-r_{b}\right)+k(1-\beta),
$$


$\Delta_{r}\left(r_{b}, r_{r}\right)=\min \left\{\Delta_{b},\left(2(1-\beta)-\left(r_{r}-r_{b}\right)\right)\right\}$,

where $k \beta<r_{b}<(k+1) \beta$ and $k=0,1$.

Proof: The proof is omitted for brevity.

Combining (25) with Lemma (IV.2), we obtain the distortion-diversity tradeoff achieved by the SRSPC scheme. We visualize the above distortiondiversity tradeoff along the direction of $\left(d_{b}, d_{r}\right)=$ $(d, \alpha d)$ for the special cases of $\alpha=1$ and $\alpha=3$ in Figures 7 and 8, respectively.

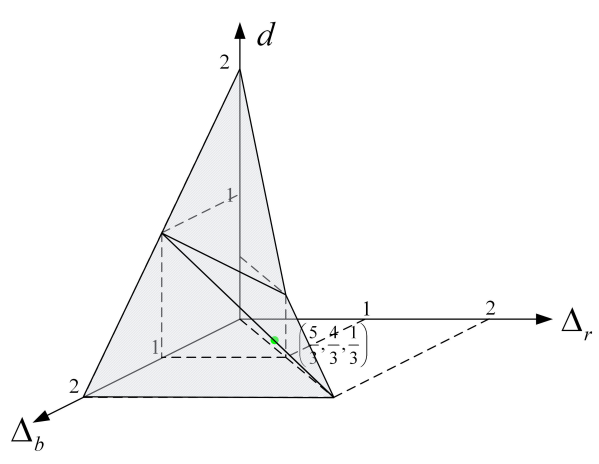

Fig. 7. Single-Block Distortion-Diversity Tradeoff of SR-SPC, $\left(d_{b}, d_{r}\right)=(d, d)$

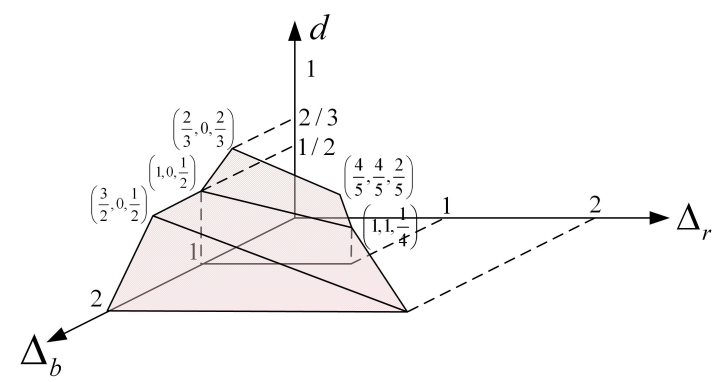

Fig. 8. Single-Block Distortion-Diversity Tradeoff of SR-SPC, $\left(d_{b}, d_{r}\right)=(d, 3 d)$

Example: green point in Figure 7 This coordinates of this point are $(5 / 3,4 / 3,1 / 3)$, which correspond to the $\alpha=1$ case. Since $d_{b}=d_{r}=1 / 3$, we assign the source/channel coding rates as follows,

$$
\begin{aligned}
& \lim _{\mathrm{SNR} \rightarrow \infty} \frac{R_{s, b}}{\ln \mathrm{SNR}}=\lim _{\mathrm{SNR} \rightarrow \infty} \frac{R_{c, b}}{\ln \mathrm{SNR}}=\frac{1}{3}, \\
& \lim _{\mathrm{SNR} \rightarrow \infty} \frac{R_{s, r}}{\ln \mathrm{SNR}}=\lim _{\mathrm{SNR} \rightarrow \infty} \frac{R_{c, r}}{\ln \mathrm{SNR}}=0 .
\end{aligned}
$$

The power allocation is SNR $-\mathrm{SNR}^{2 / 3}$ and $\mathrm{SNR}^{2 / 3}$ for the base layer and the refinement layer, respectively. The base reconstruction and the refinement reconstruction outage events (26) and (27) reduce to Figure 9. If we proceed along the direction of

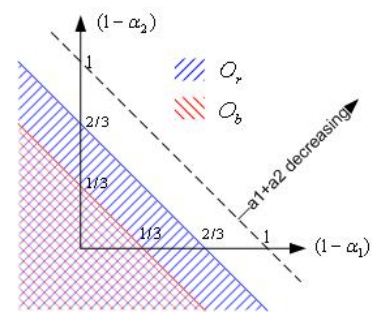

Fig. 9. Example: SR-SPC Outage Event Relationship

decreasing $\alpha_{1}+\alpha_{2}$ in 9 , we can read off the minimal $\alpha_{1}+\alpha_{2}$ of each outage event as follows,

$$
\Delta_{b}=5 / 3, \quad \Delta_{r}=4 / 3,
$$

which, combined with the fact that $d_{b}=d_{r}=$ $1 / 3$, yields the desired distortion-diversity point $\left(d_{b}, \Delta_{b}, d_{r}, \Delta_{r}\right)=(1 / 3,5 / 3,1 / 3,4 / 3)$.

\section{Performance Outer Bound}

To derive the performance outer bound, we first obtain the distortion-diversity tradeoff for singlelayer source-channel coding schemes. A block diagram of the single-layer scheme is shown in Figure 10. For the source coding part, we use a

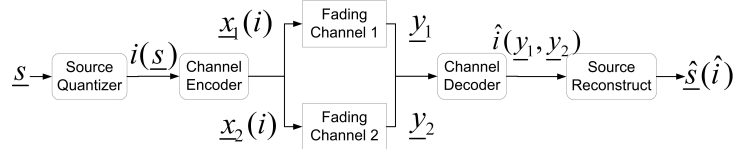

Fig. 10. Single-Layer Sour-Channel Scheme

source quantizer operating on the single description rate distortion boundary and quantizes a length- $N$ 
complex Gaussian source sequence into a single description $i \in\{1, \cdots, M\}$. For the channel coding part, we consider a random channel codebook $\mathcal{C}$ composed of $M$ space-time codewords (each codeword is a $2 \times N$ matrix, $\left(\underline{\mathbf{x}}_{1}(i), \underline{\mathbf{x}}_{2}(i)\right)$, the entries of which are i.i.d. generated according to $\mathcal{C N}(0, \mathrm{SNR}))$. The source coding rate and the channel coding rate are

$R_{s}=\frac{\ln M}{N}($ nats $/ s s), \quad R_{c}=\frac{\ln M}{N}($ nats $/ \mathrm{cu})$.

The channel decoder searches the channel codebook $\mathcal{C}$ for the codeword $\left(\underline{\mathbf{x}}_{1}(\hat{i}), \underline{\mathbf{x}}_{2}(\hat{i})\right)$ that is jointly typical with the channel output sequence pair $\left(\underline{\mathbf{y}}_{1}, \underline{\mathbf{y}}_{2}\right)$ w.r.t. the instantaneous fading coefficients.

Recall that the single-block supportable rate of the parallel fading channel (2) is,

$$
I\left(\mathbf{h}_{1}, \mathbf{h}_{2}, \mathrm{SNR}\right)=\sum_{i=1,2} \ln \left(1+\left|\mathbf{h}_{i}\right|^{2} \mathrm{SNR}\right) .
$$

Note that the supportable rate, when averaged over the fading coefficients, grows with SNR as $2 \ln$ SNR in the high SNR regime. To achieve a vanishing outage probability as SNR increases, we let the channel coding rate $R_{c}$ scale with SNR as $R_{c}=r \ln$ SNR $(0<r<2)$. The outage event is defined as follows,

$\mathcal{O}\left(\left|\mathbf{h}_{1}\right|,\left|\mathbf{h}_{2}\right|, R_{c}, \mathrm{SNR}\right)=\left\{I\left(\mathbf{h}_{1}, \mathbf{h}_{2}, \mathrm{SNR}\right)<R_{c}\right\}$.

which reduces in the high SNR regime to,

$$
\mathcal{O}^{\prime}\left(\alpha_{1}, \alpha_{2}, r\right)=\left\{\left(1-\alpha_{1}\right)^{+}+\left(1-\alpha_{2}\right)^{+}<r\right\} .
$$

Applying Lemma II.1, the diversity order of the above outage event is

$$
\Delta(r)=\Delta_{\mathcal{O}^{\prime}\left(\alpha_{1}, \alpha_{2}, r\right)}=2-r .
$$

If the outage does not happen, then the distortion level achieved by the source coding rate $R_{s}$ is

$$
D\left(R_{s}, \operatorname{SNR} \mid \mathcal{O}^{c}\right)=e^{-R_{s}}=\mathrm{SNR}^{-r},
$$

since $R_{s}=R_{c}=r \ln$ SNR. We focus on the exponentially decreasing speed of the distortion level and define the distortion exponent as

$$
d\left(R_{s}\right) \triangleq-\lim _{\mathrm{SNR} \rightarrow \infty} \frac{\ln D\left(R_{s}, \mathrm{SNR} \mid \mathcal{O}^{c}\right)}{\ln \mathrm{SNR}}
$$

which, in this case, reduces to

$$
d(r)=-\lim _{\mathrm{SNR} \rightarrow \infty} \frac{\ln \mathrm{SNR}^{-r}}{\ln \mathrm{SNR}}=r .
$$

Expressions (31) and (33) provide a parametric characterization of the tradeoff between the diversity order $\Delta$ and the distortion exponent $d$ for the single reconstruction layer,

$$
\Delta=2-d, \quad 0<d<2 .
$$

Any double-layer scheme achieves two different reconstructions, under the name high-priority reconstruction and low-priority reconstruction, respectively. Note that the partial reconstruction achieved by MDC-JD and the base reconstruction achieved by SR-SPC belong to the high-priority reconstructions, while the full reconstruction by MDCJD and the refinement reconstruction by SR-SPC belong the low-priority reconstructions. We use $\left(d_{H}, \Delta_{H}\right)$ to denote the distortion exponent and the diversity order of the high-priority reconstruction, $\left(d_{L}, \Delta_{L}\right)$ for the low-priority reconstruction. The distortion-diversity outer bound is obtained as follows: $\left(d_{H}, \Delta_{H}\right)$ is bounded by the single layer distortion-diversity boundary $\Delta_{H}=2-d_{H}$; similarly, $\left(d_{L}, \Delta_{L}\right)$ is bounded by $\Delta_{L}=2-d_{L}$; naturally, $\Delta_{H}>\Delta_{L}$, implied from their names. The fact that the single layer distortion-diversity boundary bounds the performance of both layers can be understood by treating the single layer scheme as a special case of the double layer scheme where we are concerned with the performance of only one reconstruction. The above distortiondiversity outer bound for double layer schemes is summarized as follows,

$$
\begin{aligned}
& \qquad\left\{\begin{array}{c}
\Delta_{H}=2-d_{H}, \\
\Delta_{L}=2-d_{L}, \\
0<d_{H}<d_{L}<2 .
\end{array}\right\} . \\
& \text { V. PERFORMANCE COMPARISON }
\end{aligned}
$$

\section{PERFORMANCE COMPARISON}

We compare the MDC-JD scheme, the SR-SPC scheme and the performance outer bound by overlaying their distortion-diversity regions along the direction of $\left(d_{p}, d_{f}\right)=\left(d_{b}, d_{r}\right)=\left(d_{L}, d_{H}\right)=$ $(\alpha d, d)(\alpha>1)$. The special cases of $\alpha=1$ and $\alpha=3$ are illustrated in Figures 11 and 12, respectively. 


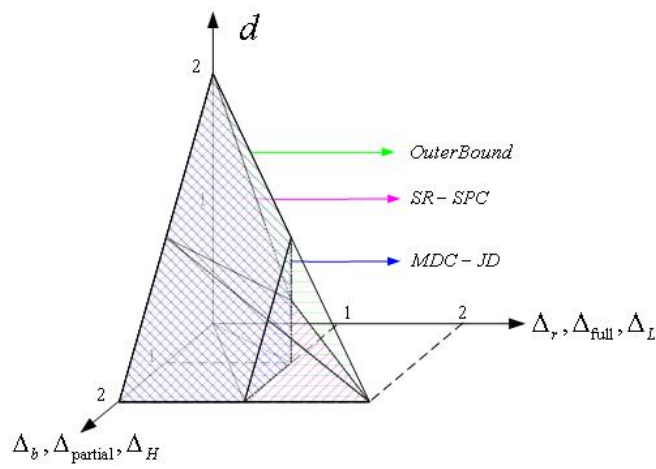

Fig. 11. Single-Block Distortion-Diversity Regions: MDC-JD, SR-SPC and Outer-Bound. $\left(d_{p}, d_{f}\right)=\left(d_{b}, d_{r}\right)=\left(d_{L}, d_{H}\right)=$ $(d, d)$

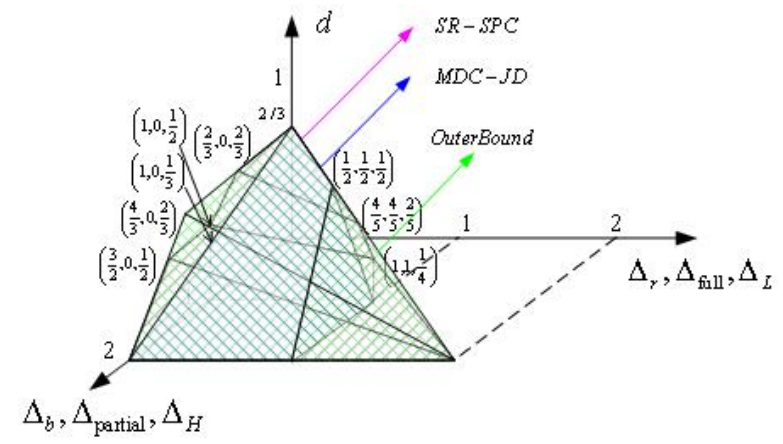

Fig. 12. Single-Block Distortion-Diversity Regions: MDC-JD, SR-SPC and Outer-Bound, $\left(d_{p}, d_{f}\right)=\left(d_{b}, d_{r}\right)=\left(d_{L}, d_{H}\right)=$ $(3 d, d)$

Remark For the $\alpha=1$ case, there is no clear winner between the MDC-JD scheme and the SRSPC scheme, as shown in Figure 11. However, for the $\alpha=3$ case, the SR-SPC scheme outperforms the MDC-JD scheme, since the MDC-JD distortiondiversity region is subsumed in the SR-SPC region, which can be observed from Figure 12.

We further illustrate the comparison of the singleblock distortion-diversity regions along the direction of $\left(d_{p}, d_{f}\right)=\left(d_{b}, d_{r}\right)=\left(d_{L}, d_{H}\right)=(\alpha d, d)$ $(\alpha>1)$ and $\left(\Delta_{\text {partial }}, \Delta_{\text {full }}\right)=\left(\Delta_{b}, \Delta_{r}\right)=$ $\left(\Delta_{H}, \Delta_{L}\right)=(\gamma \Delta, \Delta)(\gamma>1)$. We have plotted the $\Delta-d$ curves in four $(\alpha, \gamma)$ settings $\{(1,1),(1,2),(3,1),(3,2)\}$, as shown in Figure 13.

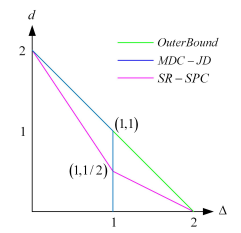

(a)

$(\alpha, \gamma)=(1,1)$

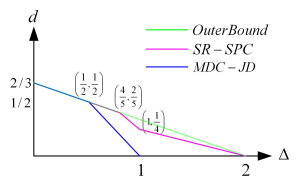

(c) $(\alpha, \gamma)=(3,1)$

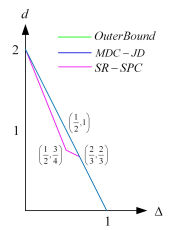

(b) $(\alpha, \gamma)=$ $(1,2)$

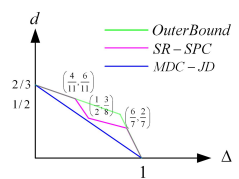

(d) $(\alpha, \gamma)=(3,2)$
Fig. 13. Single-Block Distortion-Diversity Regions: MDC-JD, SR-SPC and Outer-Bound

\section{REFERENCES}

[1] L. Zheng and D. N. C. Tse, "Diversity and Multiplexing: A Fundamental Tradeoff in Multiple Antenna Channels", IEEE Tran. on Info. Theory, vol. IT-1, no. 8, pp. 1-25, Aug. 2002.

[2] A. El Gamal and T. M. Cover, "Achievable rates for multiple descriptions". IEEE Tran. Info. Theory, vol. IT28, no. 6, pp. 851-857, Nov. 1982.

[3] W. H. R. Equitz and T. M. Cover, "Successive fefinement of information". IEEE Tran. Info. Theory, vol. IT-37, no. 2, pp 269-274, Mar. 1991.

[4] J. N. Laneman, E. Martinian, G. W. Wornell and J. G. Apostolopoulos, "Source-channel diversity for parallel channels", IEEE Tran. Info. Theory, vol. IT-51, no. 10, pp. 3518-39, Oct. 2005.

[5] S. N. Diggavi and D. N. C. Tse, "Fundamental Limits of Diversity-Embedded Codes over Fading Channels", IEEE ISIT Adelaide, Australia, pp 510-514, Sep. 2005.

[6] A. El Gamal and T. M. Cover, "Information Theory of Multiple Descriptions", presented at Shannon Theory Workshop, Mt. Kisco, NY, Sep. 1979. 